\title{
A semi-dynamic model for competition over popularity and over advertisement space in social networks
}

\author{
Eitan Altman \\ INRIA Sophia-Antipolis, 2004 Route des \\ Lucioles, 06902 Sophia-Antipolis Cedex, France \\ Email: Eitan.Altman@inria.fr \\ URL: http://www-sop.inria.fr/members/Eitan.Altman/
}

\begin{abstract}
Various tools are available for increasing the speed of content dissemination such as embeddinigs in some popular web pages, sharing in some other social networks, and advertisement. In particular, when individuals pass through a content provider to distribute contents, they can benefit from tools such as recommendation systems. The content provider can give a preferential treatment to individuals who pay for advertisement. In this paper we study competition between several contents, each characterized by some given potential popularity. We study competition through advertisements that are placed at the beginning of the dissemination of contents. We answer the question of when is it worthwhile to invest in advertisement as a function of the potential popularity of a content as well as its competing contents. The competition between similar contents (e.g. news channels) over a finite set of potential destinations. We then consider a second model in which there is also competition on advertisement space. We compute the equilibrium strategy and identify its structure and properties for each one of the situations.
\end{abstract}

\section{INTRODUCTION}

We consider in this paper competition between individuals (that we call "seeds") who create contents and wish to disseminate their content using the some social network. We assume that it is either the content that the seeds sell to interested peers or that their content provides information to some other product that peers are interested in. We assume that a seed can increase its popularity using various costly actions. We call these dissemination acceleration actions. In particular, one can propose to pay the network provider in order to receive a preferential treatment to one's content and have its rate of dissemination increased. This paper focuses on the study of acceleration actions at the beginning of the dissemination process.

We study two types of actions.

- The first are user independent actions. These are actions that several users can take, independently of each other. For example, the owner of a hotel can put an advertisement in sites specializing in proposing tourist packages.
- The second type consists of competition over an exclusive resource that can be allocated to one user. We assume that the choice to whom it will be allocated is uniformly distributed among those that propose to pay for a preferential treatment.

As an example of actions of the first kind, observe Figure 1 that concerns a video clip of Obama over Youtube. Below the screen we can see the popularity curve (in terms of number of downloads) of the content as a function of time. We can also see some initial actions that increased the popularity of the clip. For example, action D consisted of embedding the video in the WEB site of the White-House. This action brought 31,008 viewers to this video as is seen in the table below the curve.

When some video makes it to the first position in the recommendation list related to a given set of tags, then it gets a higher visibility than the others that appear in that list, and therefore the speed of propagation is expected to increase. The first position in the list is available for seeds that pay for appearing there.

As an example of the second type of actions, observe Fig 2 that shows the computer screen that I had when watching a video clip on music by Piazzola using Youtube. One can observe three types of advertisements. There is an advertisement for EFS at the bottom of the large dark rectangle which is the screen that shows the video. If one wishes to watch the video then the dark rectangle will occupy the whole computer screen and then this advertisement will be the only one you would see. There is a second advertisement at the top right part of the screen - for courses in Piano Jazz. The first two advertisements are not special for content. We are interested in a third type of advertisement: To the right we see the first five video clips in a recommendation list provided by Youtube. The first in the list has a tag "Ad". It is a video clip that received a priority in the recommendation list. Only one clip is reserved in the recommendation graph for advertisement purposes. 
President Obama Sings "Sweet Home Chicago"

\section{whitehouse Subscribe 2,720 videos -}

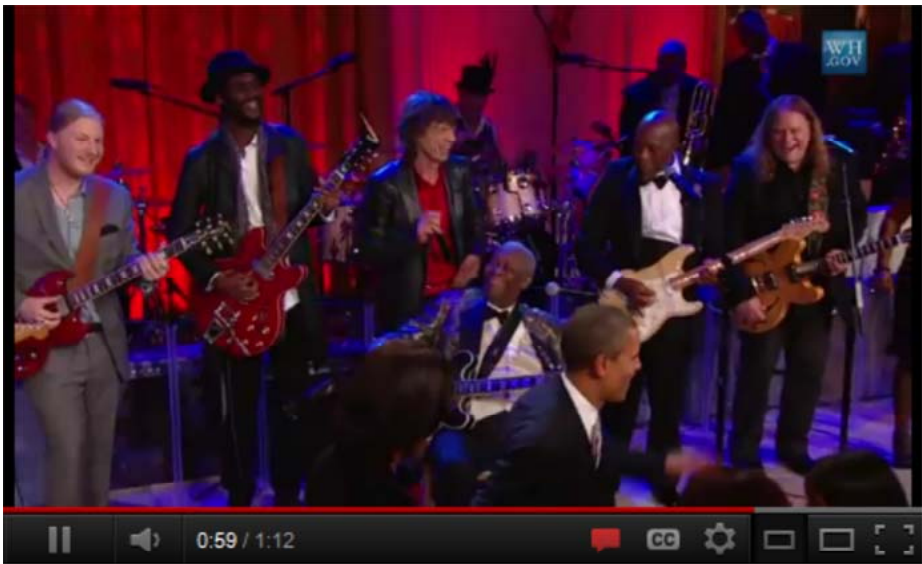

Like $\quad$ Add to $=$ Share

404,721

Total views: 404,721

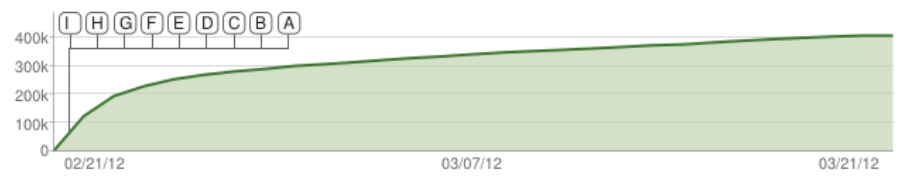

Ratings: 3452

Likes: 3237

Comments: 1,973

Dislikes: 215

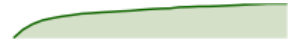

Favorites: 920

Significant discovery events

\begin{tabular}{|c|c|c|}
\hline & Date & Event \\
\hline A & $02 / 22 / 12$ & First view from a mobile device \\
\hline & $02 / 22 / 12$ & First embedded on - facebook.com \\
\hline & $02 / 22 / 12$ & First referral from YouTube search - obama singing \\
\hline & $02 / 22 / 12$ & First embedded on - whitehouse.gov \\
\hline & $02 / 22 / 12$ & First referral from - facebook.com \\
\hline & $02 / 22 / 12$ & First embedded on - plus.google.com \\
\hline & $02 / 22 / 12$ & First referral from YouTube search - barack obama singing \\
\hline & $02 / 22 / 12$ & First referral from YouTube search - obama sings \\
\hline & 02/22/12 & First embedded on - failblog.org \\
\hline
\end{tabular}

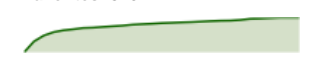

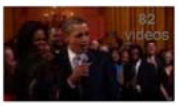

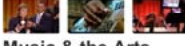 \\ Music \& the Arts}

Backstage at the White House: Tedeschi \&

by whitehouse

54.660 views

President Obama Welcomes Guests to "In by whitehouse

21,945 views

President Obama at the University of Miami

by whitehouse

First Lady Surprises

by whitehouse

89.265 views

President Obama's Bilateral Meeting with by whitehouse

3/20/12: White House Press Briefing

by whitehous

301 views

President Obama's Bilateral Meeting with

by whitehouse

305 views

On Nowruz, President Obama Speaks to the

by whitehouse

752 views

يزيدينت اوياما در نوروز با ايرائيان

صحيت كرد

by whitehouse

989 views

في عيد نوروز، الرئيس أوبالما

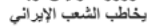

by whitehouse

301 views

3/19/12: White House

Press Briefing

by whitehouse

876 views

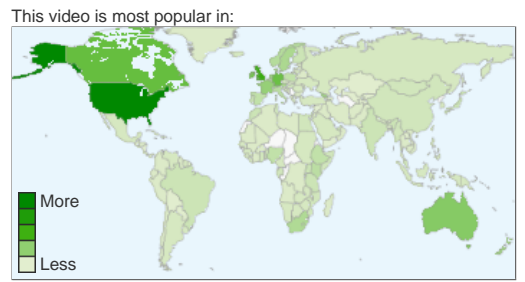

First Question with Jay Carney - March 19th 2012 by whitehouse 2.502 views

Weekly Address: Ending Subsidies for Big Oil

by whitehouse
27.982 views

Fig. 1. Actions for increasing the popularity of content: embedding and sharing 
In addition to a competition on the preferential service we consider also a competition over a common consumer population target. More precisely, we assume that there is a finite population that are potentially interested by content proposed by each one of several competing seeds. Any individual within this set will purchase the content from the first seed that it becomes aware of. Thus paying for speeding up the rate of dissemination would then allow a seed not only to reach its target population faster but also decrease the number of peers that the competitors would reach. We formulate this decision problem as a game with finite state and action spaces. The solution of the problem allows us to provide guidelines for individual's advertisement strategies.

Related work: Epidemic propagation of information has been extensively studied in the context of marketing, and various related games have been formulated and solved. See [13] for a survey as well as [8], [11].

In contrast with previous work, our models focus on decision making by individuals who create contents and who compete over consumers, and their interaction with advertisement opportunities proposed either by the owner of the social network or by other coupled social networks. In a previous related work [3], we studied a stochastic game for dynamic advertisement strategies over a social network. There, new advertising opportunities were assumed to be available at each time epoch. Various information structures were considered. The state dependent equilibrium was computed and showen to be of a threshold type under some assumptions on the cost. More precisely, it was shown that advertisement effort is larger at equilibrium for seeds of more popular contents. Moreover, it is increasing in the amount of destinations that have not yet received any content. In contrast, the present work considers two types of advertizing that occur when initiating the spreading

An optimization (rather than a game approach) for competition over advertisement space has been extensively studied within the framework of competition over shelf space, see e.g. [7] and references therein. Most work on this area do not take into account the impact of the advertisement on the dynamics of the future potential demand. Such dependence is studied in [7], where the demands are assumed to be functions of inventory sizes.

We restrict our attention in this paper to strategic interaction between similar types of content. Some initial work on the question of competition between different types of content can be found in [2]. Other aspects of competition between both service providers as well as content providers can be found in [1], [4], [6], [12], [5].

We begin by introducing in Section II a dynamic model that describes the impact of competition over the the population target on the dissemination of the competing contents. We then study in Sections III and IV the equilibria obtained in advertising games when using the two types of actions, respectively (the user independent case and the exclusive case).

\section{Modeling Dissemination IN A COMPETING ENVIRONMENT}

Assume that there is a set $\mathcal{N}$ of $N$ competing contents among the subscribers of a social network (the content may correspond to, say, some text editting softwares that are sold over the network).

Let $M$ the number of peers in the network that are interested in the content originating in $\mathcal{N}$.

We assume that opportunities for accessing a content $n$ arrive at destination $m$ according to a Poisson process with parameter $\lambda_{n}$ starting at time $t=0$. Hence if at time $t=0$ destination $m$ were interested in content $n$, it would have to wait some time which is exponentially distributed with parameter some parameter $\lambda_{i}$. We shall consider possibilities of accelerating these rates by putting some efforts, such as advertisement. In the absence of any such effort we shall have $\lambda_{i}=\phi_{i}$ where $\phi_{i}$ are some constants. $\phi_{i}$ is called the basic "popularity rate of content $i$ ".

Let $X_{i}(t)$ be the number of destinations that have obtained by time $t$ content from seed $i$. Let $x_{i}(t):=E\left[X_{i}(t)\right]$ and $x(t):=\sum_{i=1}^{N} x_{i}(t)$. Then

$$
\dot{x}_{i}(t)=\lambda_{i}(M-x(t))
$$

Taking the summation over $i$ in (1), we get,

$$
\dot{x}(t)=\lambda(M-x(t))
$$

where $\lambda=\sum_{i=1}^{N} \lambda_{i}$.

The solution of (2) is

$$
x(t)=x(0)-(M-x(0))(1-\exp (-\lambda t))
$$

Thus $x_{i}(t)$ is the solution of

$$
\dot{x}_{i}=\frac{\lambda_{i}}{\lambda} \dot{x}(t)
$$

and is given by

$$
x_{i}(t)=x_{i}(0)+\left(M \frac{\lambda_{i}}{\lambda}-x_{i}(0)\right)(1-\exp (-\lambda t))
$$

In the special case that $x_{i}(0)=0$ for all $i$, we get

$$
x_{i}(t)=M \frac{\lambda_{i}}{\lambda}(1-\exp (-\lambda t))
$$

Note, finally, that for any initial state,

$$
\lim _{t \rightarrow \infty} x_{i}(t)=M \frac{\lambda_{i}}{\lambda} .
$$

We call this the "dissemination utility".

Although the above model is quite simplistic, the form of the last equation is quite similar to the form of the curves describing the evolution of popularity in Youtube videos. As an example, observe the curve

$$
x_{i}(t)=420000(1-\exp (-0.3 t))
$$

depicted in Figure 3. It is obtained from the last equation by identifying $\lambda=0.3$ and $M \frac{\lambda_{i}}{\lambda}=420000$. With these estimations we see that the curve of number of viewers in 


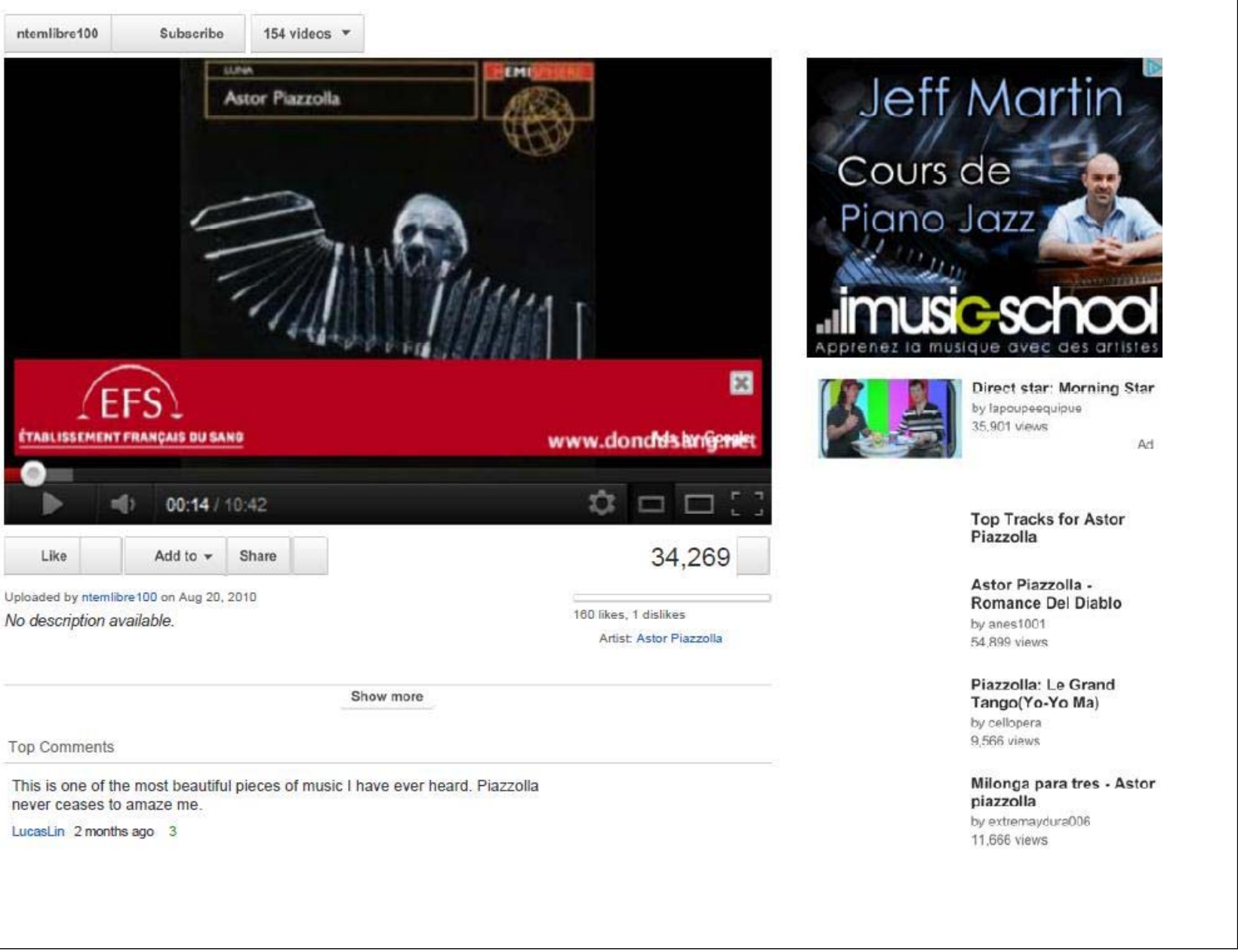

Fig. 2. Advertisements in Youtube

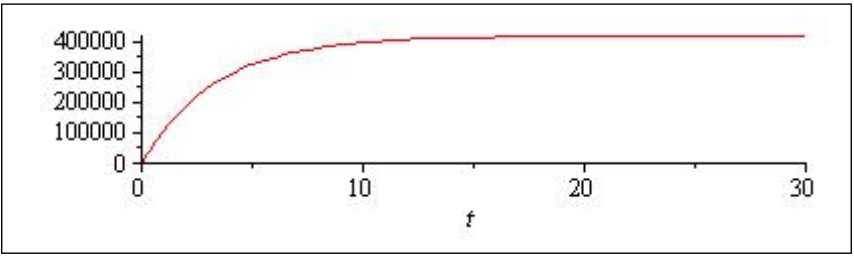

Fig. 3. A mathematical model for the evolution of the popularity of Obama

Figure 3 is quite similar to the one obtained using the youtube statistics in Figure 1.

In the next Sections we shall build on the results of this Section. We shall especially make use of the fact that as $t \rightarrow$ $\infty, x_{i}(t)$ converges (monotonically) to $x_{i}:=M \frac{\lambda_{i}}{\lambda}$.

\section{THE ADVERTISEMENT GAME OF USER INDEPENDENT ACTIONS}

Next, we assume that $\lambda_{j}$ are decision variables. Thus any player $j$ can choose $\lambda_{j}$ satisfying $\lambda_{j} \geq \phi_{j}$. Choosing the minimum value $\phi_{j}$ corresponds to deciding not to accelerate. $\lambda_{j}, j=1, \ldots, N$ is an equilibrium if for each $j, \lambda_{j}$ maximizes $M W_{j}(x)$ over $x_{j} \geq \phi_{j}$ where

$$
W_{j}(\mathbf{x}):=\frac{x_{j}}{\sum_{k} x_{k}}-\gamma\left(x_{j}-\phi_{j}\right)
$$

$\gamma M>0$ is the acceleration cost. Note that this utility function is concave in $x_{j}$.

We write the Lagrangian for player $j$ as

$$
L_{j}(\mathbf{x})=W_{j}(\mathbf{x})-\beta_{j}\left(x_{j}-\phi_{j}\right)
$$

where the Lagrange multipliers $\beta_{i}$ are non-positive.

The best response: we solve

$$
0=\frac{\partial L_{j}}{\partial x_{j}}=\frac{\sum_{n \neq j} x_{n}}{\left(\sum_{n=1}^{N} x_{n}\right)^{2}}-\gamma-\beta_{j}
$$

which gives the quadratic equation in $x_{j}$ :

$$
x_{j}^{2}+2\left(\sum_{n \neq j} x_{n}\right) x_{j}+\left(\sum_{n \neq j} x_{n}\right)^{2}-\frac{\sum_{n \neq j} x_{n}}{\gamma+\beta_{j}}=0
$$


The non-negative solution is given by

$$
x_{j}=-\sum_{n \neq j} x_{n}+\sqrt{\left(\beta_{j}+\gamma\right)^{-1}\left(\sum_{n \neq j} x_{n}\right)}
$$

Denote $\psi=\sum_{i=1}^{N} x_{i}$. Then (6) implies that for all $j$,

$$
\psi^{2}\left(\beta_{j}+\gamma\right)=\sum_{n \neq j} x_{n}=\psi-x_{j}
$$

Taking the sum over all $j$ we get the quadratic equation in $\psi$ :

$$
\psi^{2} \sum_{j}\left(\beta_{j}+\gamma\right)=(N-1) \psi
$$

so finally

$$
\psi=\frac{N-1}{\sum_{j}\left(\gamma+\beta_{j}\right)}
$$

We solve this for the symmetric case in the next subsection and then solve complete the solution for the general case.

\section{A. The symmetric case}

In the symmetric case, when $\phi=\phi_{j}$ are the same for all $j$, we have by (8)

$$
x_{j}=\max \left(\phi, \frac{N-1}{N^{2} \gamma}\right)
$$

We used here the complementarity property of the Lagrange multipliers which state that $\left(x_{i}-\psi\right) \beta_{i}=0$. This equals $\phi$ as long as

$$
\gamma \geq \frac{N-1}{N^{2} \phi}=: \gamma_{0}
$$

Assuming that the gain of the network is given by the advertisement cost, we have for any $\gamma \geq \gamma_{0}$ zero gain. For any $\gamma<\gamma_{0}$, on the other hand, the gain of the network is

$$
N \gamma\left(x_{j}-\phi\right)=N(1-\gamma \phi)-1
$$

This can be made arbitrarily close to $N$ by choosing $\gamma$ sufficiently small.

The globally (social) optimum is obtained for $x_{j}=\phi$.

The price of anarchy for a given $\gamma_{0}$ is given by 1 for $\gamma \geq \gamma_{0}$, and by $N(1-\gamma \phi)$ otherwize. Thus

$$
P o A=\max (1, N(1-\gamma \phi))
$$

which can be made arbitrarily close to $N$ by choosing $\gamma$ small enough.

\section{B. The general non-symmetrical case}

Substituting (8) in (7) we obtain

$$
x_{j}=\psi\left(1-\psi\left(\gamma+\beta_{j}\right)\right) .
$$

Due to the complementarity conditions on $\beta_{j}$, whenever $x_{j}>\phi_{j}$ then $\beta_{i}=0$.

This, together with (9) imply that for all $j$ for which $x_{j}>$ $\phi_{j}$, we have

$$
x_{j}=\psi(1-\psi \gamma)
$$

and the rest do not accelerate, i.e. $x_{j}=\phi_{j}$.

Hence there is some $\alpha$ such that $x_{i}=\phi_{i}$ for all $i$ such that $\phi_{i}>\alpha$ and for all other $i$ 's, $x_{i}$ are equal and given by (10). For all these, the equilibrium value does not depend on $\phi_{i}$. Thus the structure of the equilibrium policy is either not to accelerate, or to accelerate till some target is reached. This target is the same for all those who accelerate and does not depend directly on the propagation rates $\phi_{i}$ before accelerating. (The values $\phi_{i}$ only determine whether there will be an acceleration of the $i$ th content).

For all $j$ for which $x_{j}=\phi_{j}$ we have by (9)

$$
\gamma+\beta_{j}=\frac{1}{\psi}\left(1-\frac{\phi_{j}}{\psi}\right)
$$

In particular, assume that

$$
\frac{N-1}{N^{2} \gamma}>\max _{j=1, \ldots, N} \phi_{j} .
$$

Then

$$
x_{i}=\frac{N-1}{N^{2} \gamma}
$$

satisfies $x_{i}>\phi_{i}$ implying $\beta_{i}=0$ for all $i$. We conclude that $x_{i}$ given in (12) is the equilibrium if (11) holds.

Next assume it does not hold. Reorder the players such that $\phi_{i}$ is decreasing in $i$. Then there is a group $\mathbf{M}$ of, say $m$ players, who do not accelerate and for whom $x_{i}=\phi_{i}$.

Assume that $m$ is the largest integer such that $x_{m}=\phi_{m}$. Then from (10),

$$
\psi=(N-m) \psi(1-\psi \gamma)+Z[m]
$$

where $Z[m]=\sum_{i=1}^{m} \phi_{i}$. Thus

$$
(N-m) \gamma \psi^{2}+\psi(1-N+m)-Z[m]=0
$$

so that

$$
\begin{aligned}
& \psi[m]=\frac{1}{2(N-m) \gamma}((N-m-1)+ \\
& \left.\sqrt{(N-m-1)^{2}+4(N-m) \gamma Z[m]}\right)
\end{aligned}
$$




\section{THE CASE OF A SINGLE ADVERTISEMENT OPPORTUNITY}

We assume that the contents of the $N$ seeds appear in some ordered recommendation graph. Thanks to its position in the recommendation list, the first in the list, say the content of seed $i$, is assumed to be more visible than the others. We assume that this translates to a larger value of $\lambda_{i}$ More precisely, we consider the situation where $\lambda_{j}$ are all equal to some constant $\eta$, except for $\lambda_{i}$ which is taken to be $a$ times that value.

We assume that each seed can make a bid in order to be in the top of the list. There is a cost $c$ for bidding which is equally shared among all those that bid, and in addition, thre is a fixed cost $d$ for bidding. If no one makes any bid then all seeds are equally likely to find their content in the head of the line, and no seed has to pay anything. If $k$ seeds bid for being the first in the line then that place will be attributed to one of them with equal probabilities.

The expected utility of player $j$ is given by

$$
U_{j}=E\left[X_{j}\right]-E\left[C_{j}\right]
$$

where

$$
X_{j}=\lim _{t \rightarrow \infty} X_{j}(t)
$$

is the number of destinations that will receive the content of seed $j$, and $C_{j}$ is the cost for bidding.

Let $R(f, n)$ denote the expected number of destinations that will receive the content of the first content in the recommendation list, given that there are $n$ seeds. Denote by $y R(o, n)$ the expected number of destinations that will reeive the content of a seed other than the first one given that there are $n$ seedsd altogether. We have by equation (5):

$$
R(f, n)=\frac{a \eta M}{(n-1) \eta+a \eta}=\frac{a M}{n-1+a} .
$$

and similarly,

$$
R(o, n)=\frac{1}{n-1+a} M .
$$

Below we shall use $U_{i}(a, p)$ as the utility for player $i$ to take an action $a$ given that each other players bid with probability p. $a$ will stand for either $B$ (to bid) or for $A$ (abstain from bidding).

Theorem 1: (i) $p=0$ is an equilibrium if

$$
\frac{M}{n} \geq \frac{a M}{n-1+a}-d-c F
$$

The value at equilibrium for each player is then $M / n$.

(ii) $p=1$ is an equilibrium if

$$
\frac{M}{n}-d-\frac{c}{n} \geq \frac{M}{n-1+a} .
$$

The value at equilibrium for each player is then $M / n-d-\frac{c}{n}$. (iii) Otherwize there is a mixed equilibrium $p$ such that at equilibrium, player is indifferent between bidding or not. $p$ is thus given by the solution of $U_{i}(B, p)=U_{i}(A, p)$ where

$$
U_{i}(A, p)=(1-p)^{n-1} \frac{M}{n}+\left(1-(1-p)^{n-1}\right) \frac{M}{n-1+a}
$$

and

$$
\begin{aligned}
U_{i}(B, p)=\left[\frac{M}{n-1+a}\right]\left(1+\frac{a-1}{n p}\left(1-(1-p)^{n}\right)\right) \\
-\frac{c}{n p}\left(1-(1-p)^{n}\right)-d
\end{aligned}
$$

Proof. We have:

$U_{i}(B, 0)=\frac{M a}{n-1+a}-d-c, \quad$ and $\quad U_{i}(A, 0)=\frac{M}{n}$.

This implies (i).

For $p=1$, we have

$U_{i}(B, 1)=\frac{M}{n}-d-\frac{c}{n} \quad$ and $\quad U_{i}(A, 1)=\frac{M}{n-1+a}$.

This implies (ii).

Assume that each seed bids with probability $p$ except for player $i$. Let $\nu$ be the number of those that bid not including player $i$.

If player $i$ abstains (does not bid), then

$$
\begin{gathered}
U_{i}(A, p)=E\left[1\{\nu=0\} \frac{M}{n}+1\{\nu>0\} \frac{M}{n-1+a}\right] \\
=(1-p)^{n-1} \frac{M}{n}+\left(1-(1-p)^{n-1}\right) \frac{M}{n-1+a} .
\end{gathered}
$$

If it bids and there are $\nu$ others that bid as well, then

$$
\begin{aligned}
& U_{i}(B, p)=E\left[\frac{M}{n-1+a} \frac{\nu+a}{\nu+1}\right]-E\left[\frac{c}{1+\nu}\right]-d \\
& =E\left[\frac{M}{n-1+a}\left(1+\frac{a-1}{\nu+1}\right)\right]-E\left[\frac{c}{1+\nu}\right]-d
\end{aligned}
$$

$\nu$ has a Binomial distribution with parameter $(n-1, p)$. Thus,

$$
\begin{gathered}
\left.E\left[\frac{1}{\nu+1}\right]=\sum_{i=0}^{n-1} P(\nu=i) \frac{1}{i+1}\right] \\
=\sum_{i=0}^{n-1} \frac{(n-1) !}{i !(n-1-i) !} \frac{1}{i+1} p^{i}(1-p)^{n-1-i} \\
=\frac{1}{n p} \sum_{i=0}^{n-1} \frac{n !}{(i+1) !(n-(i+1)) !} p^{i+1}(1-p)^{n-(i+1)} \\
=\frac{1}{n p}\left(1-(1-p)^{n}\right)
\end{gathered}
$$

Taking into account the bidding costs we have

$$
\begin{aligned}
U_{i}(B, p)=\left[\frac{M}{n-1+a}\right]\left(1+\frac{a-1}{n p}\left(1-(1-p)^{n}\right)\right) \\
-\frac{c}{n p}\left(1-(1-p)^{n}\right)-d
\end{aligned}
$$

(iii) now follows since, if for some $p \in[0,1], E\left[U_{i}(A, p)\right]=$ $E\left[U_{i}(B, p)\right]$ then $p$ is an equilibrium. 


\section{CONCLUDing REMARKS}

We have presented in this paper two types of competitive interactions between content producers (seeds) who use a social network to disseminate their content. In both there is a competition over a limited common set of potential destinations. The decisions available to the seeds are related to costly advertisement and their decisions may depend on the level of popularity of their contents. In the first scenario we considered situations where each of several seeds can increase its dissemination rate by some actions such as advertisement, sharing and embedding. In the second we considered situations in which the competition is also on the limited advertisement opportunities. We characterized in both cases the equilibrium advertisement policies and identified their structure.

Our model accounts for the fact that as the game goes on, it becomes more and more difficult to further disseminate the content due to the competition over a common set of destinations. This is due to the fact that once a destination receives some content, it will not be interested in any other competing content (i.e. content produced by a competing source). This feature is related to problems of pricing of perishable or of seasonal goods in which one also has to take into consideration the fact that if a good is not sold now, it will be harder to sell it later, see [9] and references therein. We note that there is already a rich literature on advertizing over the Internet, that do not take into consideration the above dynamic aspect of the demand (e.g. [10]) but who focus on other important modeling issues.

Note that our models do not account for viewers that get to know about the existence of a given content from sources external to the network (for example from watching T.V.). If there is indeed an additional external fixed demand process for the content, then one can expect the number of viewers not to converge to a fixed constant $M$ but instead, to have asymptotically a constant increase rate. This may explain the slight deviations of the curve in Figure 3 with respect to that of 1. In fact a better fit to the curve in the upper part of Fig 4 is obtained by adding such a constant demand; this gives the low part of Fig. 4. It is seen to approximate well the curve which is given by YouTube. We shall study such models in the future.

\section{ACKNOWLEDGEMENT}

This work was partly supported by the Congas European Project.

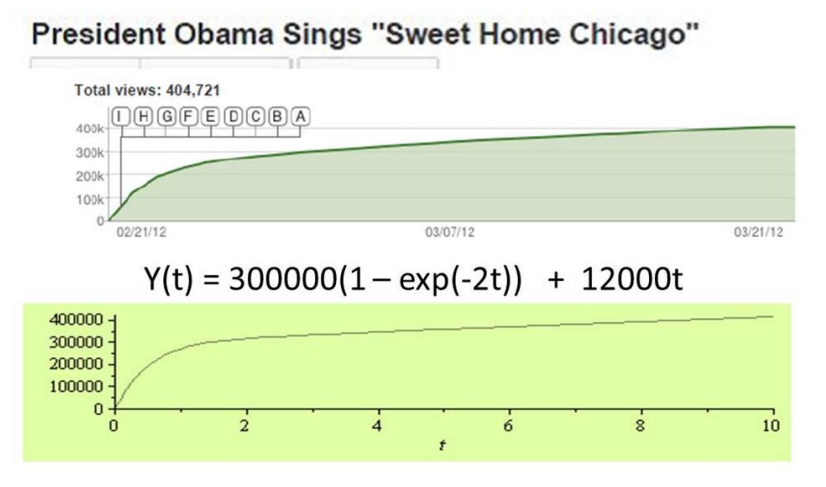

Fig. 4. An improved mathematical model (the low part of the figure) for the evolution of the popularity of the number of viewers of Obama (given in the upper part of this curve)

\section{REFERENCES}

[1] Tania Jimenez aand Yezekael Hayel and Eitan Altman. In which content to specialize? a game theoretic analysis. In Networking 2012 Workshops. LNCS, volume 7291. Springer, Heidelberg, 2012.

[2] Eitan Altman. In which content to specialize? a game theoretic analysis. In Networking 2012 Workshops. LNCS, volume 7291. Springer, Heidelberg, 2012.

[3] Eitan Altman. A stochastic game approach for competition over popularity in social networks. submitted to Dynamic Games and Applications, available at http://hal.inria.fr/hal-00681959, 2012.

[4] Eitan Altman, Pierre Bernhard, Stéphane Caron, George Kesidis, Julio Rojas-Mora, and Sulan Wong. A study of non-neutral networks with usage-based prices. In ETM, pages 76-84, 2010.

[5] Eitan Altman, Arnaud Legout, and Yuedong Xu. Network non-neutrality debate: An economic analysis. In Networking (2), pages 68-81, 2011.

[6] Eitan Altman, Sulan Wong, and Julio Rojas-Mora. P2p business and legal models for increasing accessibility to popular culture. In DigiBiz, pages 130-138, 2009.

[7] Opher Baron, Oded Berman, and David Perry. Items competing over shelf space when demand depends on inventory level. Production and Operations Management, 20(5):714-726, 2011.

[8] Marcus Chi-Hung Ling and Kevin Lawler. Internet advertising, game theory and consumer welfare. Electronic Commerce Research, 1(1-2), 2001.

[9] A. Karpowicz and K. Szajowski. Double optimal stopping times and dynamic pricing problem: description of the mathematical model. Math. Methods Oper. Res., 66(2):235-253, 2007.

[10] John Langford, Lihong Li, Yevgeniy Vorobeychik, and Jennifer Wortman. Maintaining equilibria during exploration in sponsored search auctions. Algorithmica, 58(4):990-1021, 2010.

[11] Rui Wang, Yongsheng Jin, and Feng Li. A review of microblogging marketing based on the complex network theory. In International Conference in Electrics, Communication and Automatic Control, pages 1053-1060, 2012.

[12] Sulan Wong, Eitan Altman, and Julio Rojas-Mora. Internet access: Where law, economy, culture and technology meet. Computer Networks, 55(2):470-479, 2011.

[13] Jennifer Wortman. Viral marketing and the diffusion of trends on social networks. Technical Report MS-CIS-08-19, Department of Computer and Information Science, University of Pennsylvania, May 152008. 